dentistry. One scholarship and two bursaries in biology were awarded, and four senior awards made in sociology. Under the schemes for the Dominions and Colonies there were eleven awards in medicine, fourteen in natural science (including one in engineering) and seven in the humanities and social sciences, in addition to ten Canadian travel grants and twelve farming scholarships (three for Africans). Two travelling fellowships were given to Indian Civil servants and fifteen Royal Society and Nuffield Foundation Commonwealth bursaries were awarded, while 124 grants were made from the Elizabeth Nuffield Educational Fund covering a wide range of projected careers.

\title{
EFFECTS OF IONIZING RADIATION ON ORGANIC AND BIOLOGICAL SOLIDS
}

$\mathrm{T}$ HE Société de Chimie Physique and the British Biophysical Society combined during July 3-4, in the Laboratoire de Chimie Physique, 11 rue Pierre Curie, Paris, to hold an informal discussion on the effects of radiation on organic and biological solids.

Opening the meeting on the first day, Prof. R. L. Platzman (Argonne National Laboratory, Illinois) outlined very clearly the present state of the theory of primary processes, and the recent developments due to Prof. U. Fano. In systems containing strongly interacting atoms, interaction may influence the primary energy absorption. Molecular structure, and particularly the presence of $\pi$-electrons, may affect the proportion of energy absorbed by different components in the primary process. In simple molecules, for example, methane, there is no evidence for such selective mechanism. More information is needed, in particular about the influence of water molecules, before this theory can be applied to biological materials.

Prof. M. Magat (Laboratoire de Chimie Physique, Paris) reviewed reactions in the solid state, in particular low-temperature radiation-induced changes such as polymerization, radical transfer and aromatic ring-opening, which occur by free-radical mechanisms. Members of the Paris group dealt in detail with these reactions, for example, Dr. E. Migirdycian and Dr. S. Ieach showed that benzene, if irradiated in the solid state, de-cyclizes and adds on aliphatic groups derived from the ethanol with which it is mixed in solid solution. Similar ring-opening was reported by Dr. K. Hyashi and Dr. S. Okamura (Osaka Laboratories, Japanese Association for Radiation Research on Polymers, Osaka) in the case of lactones, when it is followed by linear polymerization, and favoured by a well-ordered crystalline state.

A feature of the meeting was the use of electranspin resonance to identify and measure the free radicals produced by irradiation. Drs. R. Bensasson, C. Chachaty, M. Bodard and R. Marx (Laboratoire de Chimie Physique, Paris) used the technique to establish the free-radical nature of low-temperature radiopolymerization of such monomers as acrylonitrile, and Dr. H. Szwarc (Institut do Chimie Physique, Paris) used it effectively to demonstrate the rapid recombination of radicals which occurs on heating irradiated cyclohexane through its transition point at $186^{\circ} \mathrm{K}$. However, other factors may be involved, apart from a phase-change, in facilitating radical reactions in the solid. Dr. E. Collinson and Prof. F. S. Dainton (University of Leeds), while demonstrating that scavenging of radicals is more effective in the solid and with aromatic compounds, conjectured that this could be due to the $\pi$-electron configuration of the phenyl groups. They also found that a glassy solid behaves more like a liquid than a crystalline solid. The important distinction between aromatic and aliphatic compounds was further emphasized in work by Dr. R. Mason (Imperial College of Science and Technology, London) on post-irradiation thermo-luminescence, when the intensity of emission was found to be a thousand-fold greater in aromatic than in aliphatic amino-acids.

Prof. J. A. V. Butler (Chester Beatty Research Institute, London) introduced the second session by stressing the need for a re-appraisal of such terms as 'direct' and 'indirect' effect, 'target', etc., and emphas ized that in living systems, the balance is dynamic, and that radiation need be damaging at only one of a large number of places.

A question of continuing interest is the role of oxygen in radiation damage, and a number of contributions dealt with this factor. Drs. A. Müller, G. Hotz, and K. G. Zimmer (Institute of Radiobiology, Nuclear Research Centre, Karlsruhe) found a modified electron-spin resonance signal when bacteriophage was pressed into pellets in such a way as to expeloxygen, and Dr. L. Ehrenberg (Institutionen för organisk kemi och biokemi, University of Stockholm) on irradiating Agrostis stolonifera seeds could distinguish two types of electron-spin resonance signal, one of which is closely correlated with oxygen, and is decreased by pre-heating, that is, when oxygen is expelled. Dr. E. L. Powers (Argonne National Laboratory, Illinois) described recent work on bacterial spores, which offer a convenient experimental system for radiation work. Part of the damage is toxic only in the presence of oxygen. This can be estimated directly, and it is also possible to observe the effect of heat by using high linear energy transfer radiation, when annealing occurs during irradiation. The heat generated during heavy-ion bombardment may be an important and neglected factor, and in the work of Dr. T. Brustad (Norsk Hydro Institute for Cancer Research, Oslo), to be mentioned later, may in part explain the apparent lack of effect of additives.

The nature of the increased damage brought about by oxygen is much under discussion, and whereas the signals observed by Dr. L. Ehrenberg are not characteristic of peroxy-formation, Prof. G. Stein, (Hebrew University, Jerusalem) found a signal when polymethylmethacrylate was irradiated in the presence of oxygen which appears to be peroxidic. This system, moreover, provides a convenient method of measuring rates of oxygen diffusion. In certain circumstances, the influence of oxygen may be minimized by limited diffusion; for example, A. B. Robins and Prof. Butler (Chester Beatty Research Institute, London) found that a high dose-rate can obscure the oxygen effect completely in the case of trypsin. 
The use of electron-spin resonance in free-radical investigations has already been mentioned, and Prof. D. J. E. Ingram (University College, Keele) outlined how this can be used in the study of proteins, where electron trapping may occur. It may be possible to correlate denaturation of a protein with an interference in this trapping process since the electron-spin resonance signal does not disappear on warming in the normal way. However, it must be remembered that when applying electron-spin resonance to biological materials, water, for example, plays a part which is little understood.

Sulphur compounds are of much interest on account of their use as protective agents. Dr. R. Koch (Freiburg) investigated more than a hundred compounds by electron-spin resonance, but no simple correlations between structure and function can be seen at the present time. The radical nature is considered to be localized on the sulphur atom, and Dr. P. Alexander (Chester Beatty Research Institute, London) and Mr. M. G. Ormerod (Royal Military College of Science, Shrivenham) provided evidence that in the case of nucleoprotein protected by cysteamine, such localization can occur, minimizing the damage. There seems little doubt that the radicals produced on irradiation of keratin are cystine-like (Dr. A. Redhardt and Prof. B. Rajewsky, Max Planck Institute for Biophysics, Frankfurt), but unaccountably, cysteine and related compounds do not appear to protect by a factor of more than two in a system such as yeast, when judged by quenching of electron-spin resonance signals (Dr. B. Smaller, Argonne National Laboratory, Illinois).

In general, it appears that dry solids are more sensitive than wet solids or solutions, and Dr. R. A. Braams (Physical Laboratory, University of Utrecht) reported a ratio of three between dry and wet tendon. Dr. Robins and Prof. Butler found solid trypsin to be more sensitive than its solutions, possibly due to the self-annealing processes in the presence of water. The last-mentioned authors also reported that the radiosensitivity of trypsin is modified by the medium from which it is evaporated and a low $p H$ leads to a highly sensitive state, possibly by aggregation. It is significant that at high linear enorgy transfer Brustad obtained target sizes in excess of the accepted molecular weight for some enzymes; this also raises the question of energy transfer in the solid state. The effect of added materials depends on their being intimately mixed with the substrate, and the state of the solid is thus again seen to be important.

Two papers were presented dealing particularly with chemical changes on irradiation. Dr. W. M. Garrison (Crocker Laboratory, Lawrence Radiation Laboratory, Berkeley) found good evidence for the conversion of the - NH-CH- configuration in amino-reids into keto-acids, and as little as $10^{-8}$ mole can be detected as derivatives. Dr. H. A. Dieu (Laboratoire de Chimie Physique, Université de Liège) pointed out that irradiation of ovalbumin leads to a more ready attack by proteolytic enzymes, and an 'unmasking' of sulphydryl groups with the heaviest doses. Moreover, since the rate of subsequent attack by pepsin depends on the dose, and that by trypsin does not, it is probable that the aromatic amino-acids have been preferentially destroyed. In the myoglobin molecule it appears that possibly the leucine residues are the foci of damage, in particular those in more-exposed parts of the molecule, and Mr. C. C. F. Blake and Dr. D. C. Phillips (Davy Faraday Laboratory, Royal Institution, London) provided evidence for this in which the effect of radiation was studied directly by X-ray crystallography.

In summing up, Dr. L. H. Gray (Radiobiology Unit, Mount Vernon Hospital, London) stressed that physical theories, such as that of Prof. U. Fano, must be cautiously applied to biological systems, bearing in mind their complexity and such unknown factors as water content. It appears that the degree of crystallinity of the solid phase may be of great importance, and electron-spin resonance has helped to emphasize this. Concerning the role of oxygen, the accessibility of this gas may be a factor that has not received enough attention hitherto.

Finally, I was struck by the different interpretations that were placed on electron-spin resonance signals, and feel that this useful technique needs to be used with reserve when drawing conclusions.

A. B. Robins

\section{BACTERIA OF THE INTESTINE}

$T_{\mathrm{H}}^{\mathrm{h}}$ HE summer conference of the Society for Applied Bacteriology was held at Wye College, Ashford, Kent, during July 11-13, and included a symposium on "Bacteria of the Intestine".

Dr. H. Williams Smith, in his paper entitled "The bacterial flora of the fæces of animals with particular reference to its development in the young", described experiments on the development of normal flora in healthy animals. Rectal swabs were shaken in phosphate buffer, and after passing the suspension through muslin, colony counts of Escherichia coli, staphylococci and Clostridium welchii were made.

Calves, lambs, piglets and a baby were examined daily for 2-3 weeks, weekly for three weeks, monthly for nine months and twelve months (baby). One rabbit was examined weekly.

In the calf, $E$. coli, streptococei and $C l$. welchit predominated on the first day, but bacteroides domination began at two days and these organisms rapidly multiplied up to fourteen days, when lactobacilli followed. Counts slowly dropped to $10^{7}-$ $10^{6} / \mathrm{gm}$. of fæces after thirty weeks.

Similar results were obtained from lambs and piglets, except that streptococci dominated later with $\boldsymbol{E}$. coli in lambs, and the piglet flora was dominated ultimately by lactobacilli. Results for the baby were similar also, with counts of $10^{11}$ for $E$. coli at first, followed by a bacteroides count of $10^{10} ; \mathrm{Cl}$. welchii rose sharply at twenty-five weeks when the child started eating solid food. The counts remained high after a year; this was different from the animals. Staph. aureus, $10^{2}-10^{6} / \mathrm{gm}$, was frequently found in the baby but not in the animals. The rabbit showed a constant bacteroides count of $10^{\circ}$; the lactobacilli and streptococcal counts were $10^{4}$ and $10^{5}$. Gram-negative anaerobic cocci up to $10^{8}-10^{9}$, and yeasts in older calves, were also found. There was a constant population of $E$. coli but frequent ehanges in phage types. 